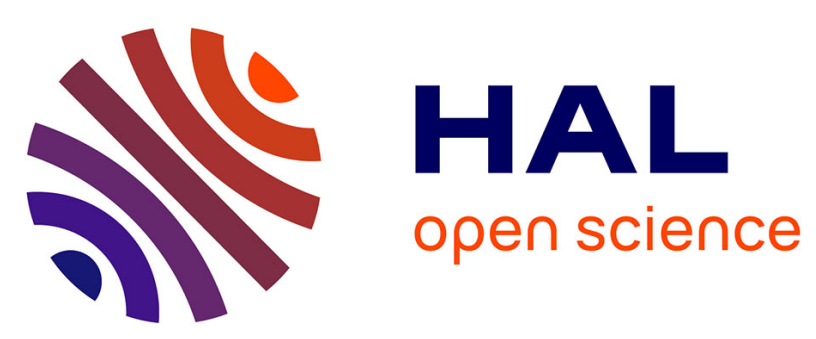

\title{
Guaranteed cost control design for synchronization in networks of linear singularly perturbed systems
}

\author{
Jihene Ben Rejeb, Irinel-Constantin Morarescu, Jamal Daafouz
}

\section{To cite this version:}

Jihene Ben Rejeb, Irinel-Constantin Morarescu, Jamal Daafouz. Guaranteed cost control design for synchronization in networks of linear singularly perturbed systems. 56th IEEE Conference on Decision and Control, CDC 2017, Dec 2017, Melbourne, Australia. hal-01653078

\section{HAL Id: hal-01653078 https://hal.science/hal-01653078}

Submitted on 1 Dec 2017

HAL is a multi-disciplinary open access archive for the deposit and dissemination of scientific research documents, whether they are published or not. The documents may come from teaching and research institutions in France or abroad, or from public or private research centers.
L'archive ouverte pluridisciplinaire HAL, est destinée au dépôt et à la diffusion de documents scientifiques de niveau recherche, publiés ou non, émanant des établissements d'enseignement et de recherche français ou étrangers, des laboratoires publics ou privés. 


\title{
Guaranteed cost control design for synchronization in networks of linear singularly perturbed systems
}

\author{
Jihene Ben Rejeb, Irinel-Constantin Morărescu and Jamal Daafouz
}

\begin{abstract}
This work presents the design of a decentralized control strategy that allows singularly perturbed multi-agent systems to achieve synchronization with global performance guarantees. The study is mainly motivated by the presence of two features that characterize many physical systems. The first is the complexity in terms of interconnected subsystems and the second is that each subsystem involves processes evolving on different time-scales. The main difficulty that we have to overcome is that we have to avoid the use of centralized information related to the interconnection network structure. This problem is solved by rewriting the synchronization problem in terms of stabilization of a singularly perturbed uncertain linear system. The singularly perturbed dynamics of subsystems generates theoretical challenges related to the stabilizing controller design but also numerical issues related to the computation of the controller gains. We show that these problems can be solved by decoupling the slow and fast dynamics. Our theoretical developments are illustrated by some numerical examples.

Index Terms-Multiagent systems; consensus; singularly perturbed systems.
\end{abstract}

\section{INTRODUCTION}

The multi-agent formalism allows treating problems coming from a wide application domain such as engineering [1], biology, sociology [2], [3], economy. Consensus and synchronization are challenging problems widely studied in the context of linear agents interacting through a directed or undirected graph with a fixed or dynamically changing topology. There are also contributions related to nonlinear agents such as oscillators dynamics [4], nonholonomic robots [1] or general nonlinear systems [5].

On the other hand, there are many applications ranging from biological systems such as gene expression systems [6], neurons behavior [7] to engineering problems [8] that involve processes evolving on different time-scales. One can find many contributions with general stability and stabilization results for such linear and nonlinear multiple time scale systems (see [9] and references therein). One of the most common approaches is the time-scale decomposition that leads to decoupled slow and fast subsystems and an appropriate combination of the corresponding results yields a control design for the original system. Many results are based on singular perturbation theory and these dynamical

This work was funded by the ANR project COMPACS - "Computation Aware Control Systems", ANR-13-BS03-004.

J. Ben Rejeb, I.-C. Morărescu, J.Daafouz are with Université de Lorraine, CRAN, UMR 7039 and CNRS, CRAN, UMR 7039, 2 Avenue de la Forêt de Haye, Vandœuvre-lès-Nancy, France. e-mails: jihene.ben-rejeb@univ-lorraine.fr, constantin.morarescu@univ-lorraine.fr, jamal.daafouzeuniv-lorraine.fr systems are also called singularly perturbed systems. The problem of synchronization of singularly perturbed systems is very challenging from a theoretical point of view and is mainly motivated by two features that characterize the nowadays systems. The first one is the complexity in terms of subsystems interconnected together in order to accomplish a global goal while the second is that physical subsystems often involve processes that evolve on different time-scales. Generally these features are tackled independently one from another.

The main goal of this paper is to design a decentralized control strategy that allows singularly perturbed multi-agent systems to achieve synchronization with global performance guarantees. Decentralized coordination control of multiagent systems attracted a lot of attention during the last decade. An important feature of this class of large scale systems is the fact that local information plays a key role. This means that each system is able to design its own control law without the help of a central entity that requires important amounts of communication and computation. Consequently, decentralized control aims at reducing the communication and computation costs. When these costs are neglected the centralized control strategies generally outperform the decentralized ones. However, energy aware strategies have to take into account the overall cost and should reduce the communication and computation loads [10]. Therefore, in this paper we design decentralized controllers that provide a guaranteed cost in the context of multi-agent systems with multiple time scale.

In a preliminary work [11], we have combined the two features presented above to study the synchronization of singularly perturbed systems. In that work we have designed decentralized controllers able to achieve asymptotically the synchronization goal. Here, we address the synchronization with global performance guarantees. One may find in the literature performance based results but in the context of multi-agent systems or separately in the context of singularly perturbed systems. Despite the interest of these contributions, synchronization of singularly perturbed systems with performance objectives is an open problem.

The contribution of this paper is the decentralized guaranteed cost control design for a multi-agent system under fixed undirected interaction graph. The dynamics of each agent is represented by linear singularly perturbed system. To solve the problem of decentralized guaranteed 
cost control design, we transform the synchronization problem in an uncertain system stabilization one. The uncertainty comes from the fact that the graph Laplacian eigenvalues are modeled as unknown but bounded uncertain parameters in order to avoid an explicit use of Laplacian's eigenvalues. This is motivated by the fact that the only available graph information consists in its connectivity. The main difficulty that we face is related to the fact that we have to ensure bounds on a global cost in a decentralized manner i.e. by a local design. Indeed, when we deal with optimal decentralized control, the Riccati equation, which, in the LQ case is the basis for the derivation of the optimal control law, involves the eigenvalues of the graph Laplacian describing the overall network. In order to get rid of this centralized information, instead of looking for an optimal controller, a guaranteed cost controller is designed to ensure a performance level of the closed-loop dynamics.

The paper is organized as follows. Section II presents some basic definitions and notations in graph theory. Section III is devoted to the design of the decentralized guaranteed cost control law. Conditions on the gain matrix such that the closed-loop singularly perturbed systems achieve asymptotic synchronization while an upper bound on the performance index is minimized, are expressed through linear matrix inequalities (LMIs). Simulation results are presented in Section IV. The paper ends with some concluding remarks.

\section{Notation}

The following standard notation is used throughout the paper. $\mathbb{R}$ is the set of real numbers, $\|x\|$ is the Euclidean norm of the vector $x$ and $\otimes$ denotes the Kronecker product of two matrices. We also denote by $I_{n} \in \mathbb{R}^{n \times n}$ the identity matrix of size $n$ and by $\mathbf{1}_{n}, \mathbf{0}_{n} \in \mathbb{R}^{n}$ the column vector whose components are all 1 and 0 , respectively. By $\mathbf{0}_{n \times m} \in \mathbb{R}^{n \times m}$ we denote the matrix whose all components are 0 . Given a matrix $A \in \mathbb{R}^{n \times n}$ and $A>\mathbf{0}(A \geq \mathbf{0})$ means that $A$ is positive (semi-) definite. The transpose of $A$ is denoted by $A^{\top}$. We denote $\operatorname{diag}\left(A_{1}, \ldots, A_{n}\right)$ the block diagonal matrix having the matrices $A_{1}$ to $A_{n}$ on the diagonal and 0 everywhere else.

\section{PRELIMINARIES AND PROBLEM FORMULATION}

We consider a network of $n$ identical singularly perturbed linear systems. The analysis is based on some graph theoretical tools that are briefly summarized in this section.

\section{A. Basic concepts of graph theory}

With the network of $n$ systems we associate a graph $\mathcal{G}$ which is a couple $(\mathcal{V}, \mathcal{E})$. Here, $\mathcal{V}=\{1, \ldots, n\}$ represents the vertex set and $\mathcal{E} \subset \mathcal{V} \times \mathcal{V}$ is the edge set. In the sequel we suppose that the graph is undirected meaning that $(i, j) \in$ $\mathcal{E} \Leftrightarrow(j, i) \in \mathcal{E}$. We also assume that $\mathcal{G}$ has no self-loop (i.e. $\forall i=1, \ldots, n$ one has $(i, i) \notin \mathcal{E})$. A weighted adjacency matrix associated with $\mathcal{G}$ is $G=\left[g_{i j}\right] \in \mathbb{R}^{n \times n}$ such that

$$
\left\{\begin{array}{l}
g_{i j}=g_{j i}>0 \text { if }(i, j) \in \mathcal{E} \\
g_{i j}=0 \text { otherwise }
\end{array} .\right.
$$

The corresponding weighted Laplacian matrix is $L=$ $\left[l_{i j}\right] \in \mathbb{R}^{n \times n}$ defined by

$$
\left\{\begin{array}{l}
l_{i i}=\sum_{j=1}^{n} g_{i j}, \forall i=1, \ldots, n \\
l_{i j}=-g_{i j} \text { if } i \neq j
\end{array}\right.
$$

By definition $L$ is symmetric and all of its rows sums are zero. Throughout the rest of the paper the following hypothesis holds.

Assumption 1: The undirected graph $\mathcal{G}$ is connected and all the non-zero weights $g_{i, j} \neq 0$ of the associated weighted Laplacian matrix are within the interval $\left[g_{m}, g_{M}\right]$ with $g_{M}>$ $g_{m}>0$.

This hypothesis is used to get consensus independently of the initial condition under consideration and to uniformly lower-bound the second smallest eigenvalue of the graph $\mathcal{G}$ as in the next Remark.

Remark 1 (Basic properties of the Laplacian matrix [12]): Let an undirected graph $\mathcal{G}$ that satisfies Assumption 1 and let $0=\lambda_{1}<\lambda_{2} \leqslant \ldots \leqslant \lambda_{n}$ be the eigenvalues of the corresponding Laplacian matrix $L$. A rough lower-bound on $\lambda_{2}$, independent of $\mathcal{G}$, is $\lambda^{*}=\frac{g_{m}^{2}}{2(n-1) n^{2}}$ (see [13] for details). Therefore, one has

$$
\lambda^{*}<\lambda_{2} \leqslant \ldots \leqslant \lambda_{n}<n \cdot g_{M} \triangleq \lambda^{\circ} .
$$

It is worth noting that there exists an orthonormal matrix $T \in \mathbb{R}^{n \times n}$ (i.e. $T T^{\top}=T^{\top} T=I_{n}$ ) such that

$$
T L T^{\top}=D=\operatorname{diag}\left(\lambda_{1}, \lambda_{2}, \ldots, \lambda_{n}\right)
$$

B. Decentralized synchronization of singularly perturbed systems

In this paper we assume that for any $i=1, \ldots, n$, the $i^{t h}$ system at time $t$ is characterized by the state $\left(x_{i}(t), z_{i}(t)\right) \in$ $\mathbb{R}^{n_{x}+n_{z}}$ and a small $\varepsilon>0$ such that its dynamics is given by:

$$
\left\{\begin{array}{rl}
\dot{x}_{i}(t) & =A_{11} x_{i}(t)+A_{12} z_{i}(t)+B_{1} u_{i}(t) \\
\varepsilon \dot{z}_{i}(t) & =A_{21} x_{i}(t)+A_{22} z_{i}(t)+B_{2} u_{i}(t)
\end{array},\right.
$$

where $u_{i} \in \mathbb{R}^{m}$ is the control input and

$$
\begin{array}{lll}
A_{11} \in \mathbb{R}^{n_{x} \times n_{x}}, & A_{12} \in \mathbb{R}^{n_{x} \times n_{z}}, \quad B_{1} \in \mathbb{R}^{n_{x} \times m}, \\
A_{21} \in \mathbb{R}^{n_{z} \times n_{x}}, & A_{22} \in \mathbb{R}^{n_{z} \times n_{z}}, & B_{2} \in \mathbb{R}^{n_{z} \times m}
\end{array}
$$

such that $\operatorname{rank}\left(B_{1}\right)=\operatorname{rank}\left(B_{2}\right)=m$.

Assumption 2: The matrix $A_{22}$ is invertible.

Definition 1: The $n$ singularly perturbed systems defined by (1) achieve asymptotic synchronization using local information if there exists a state feedback controller of the form

$$
\begin{aligned}
& u_{i}(t)=K_{1} \sum_{j=1}^{n} g_{i j}\left(x_{i}(t)-x_{j}(t)\right)+ \\
& +K_{2} \sum_{j=1}^{n} g_{i j}\left(z_{i}(t)-z_{j}(t)\right), K_{1} \in \mathbb{R}^{m \times n_{x}}, K_{2} \in \mathbb{R}^{m \times n_{z}}
\end{aligned}
$$

such that

$$
\lim _{t \rightarrow \infty}\left\|x_{i}(t)-x_{j}(t)\right\|=0 \text { and } \lim _{t \rightarrow \infty}\left\|z_{i}(t)-z_{j}(t)\right\|=0 .
$$


Let $x(t)=\left(x_{1}(t)^{\top}, \ldots, x_{n}(t)^{\top}\right)^{\top} \in \mathbb{R}^{n \cdot n_{x}}$ and $z(t)=$ $\left(z_{1}(t)^{\top}, \ldots, z_{n}(t)^{\top}\right)^{\top} \in \mathbb{R}^{n \cdot n_{z}}$ be the vectors collecting the individual states $x_{i}(t)$ and $z_{i}(t)$ of the $n$ agents. Let also $u(t) \in \mathbb{R}^{n \cdot m}$ be the vector collecting the individual controllers $u_{i}(t), i=1, \ldots, n$ (i.e $u(t)=$ $\left.\left(u_{1}(t)^{\top}, \ldots, u_{n}(t)^{\top}\right)^{\top}\right)$. Finally, let us consider the following global cost associated with synchronization of the dynamics in (1):

$$
\begin{gathered}
J=\int_{0}^{\infty} x(t)^{\top}\left(L \otimes I_{n_{x}}\right) x(t)+z(t)^{\top}\left(L \otimes I_{n_{z}}\right) z(t) \\
+u(t)^{\top}\left(I_{n} \otimes R\right) u(t) d t
\end{gathered}
$$

where $R \in \mathbb{R}^{m \times m}$ is a positive definite matrix that penalizes the control effort required for synchronization.

\section{GuARAnTEED COST CONTROL DESIGN}

\section{A. Preliminaries}

Let us recall that in [11] we used the change of variables

$$
\tilde{x}(t)=\left(T \otimes I_{n_{x}}\right) x(t), \quad \tilde{z}(t)=\left(T \otimes I_{n_{z}}\right) z(t)
$$

to rewrite the synchronization problem related to systems (1) as a simultaneous stabilization problem for systems

$$
\left\{\begin{array}{c}
\dot{\tilde{x}}_{i}(t)=\left(A_{11}-\lambda_{i} B_{1} K_{1}\right) \tilde{x}_{i}(t)+\left(A_{12}-\lambda_{i} B_{1} K_{2}\right) \tilde{z}_{i}(t) \\
\varepsilon \dot{\tilde{z}}_{i}(t)=\left(A_{21}-\lambda_{i} B_{2} K_{1}\right) \tilde{x}_{i}(t)+\left(A_{22}-\lambda_{i} B_{2} K_{2}\right) \tilde{z}_{i}(t) \\
i=2, \ldots, n
\end{array}\right.
$$

The design of $K_{1}$ and $K_{2}$ solving the simultaneous stabilization of (5) is based on the classical singular perturbation design (see [9] for instance) i.e. introducing the reducedorder systems

$$
\left\{\begin{array}{c}
\dot{\tilde{x}}_{i, s}(t)=\left(A_{0}-\lambda_{i} B_{0} K_{0}\right) \tilde{x}_{i, s}(t), \quad \tilde{x}_{i, s}(0)=\tilde{x}_{i}(0) \\
\tilde{z}_{i, s}(t)=-A_{22}^{-1}\left(A_{21}-\lambda_{i} B_{2} K_{0}\right) \tilde{x}_{i, s}(t) \\
i=1, \ldots, n
\end{array}\right.
$$

and the corresponding boundary layer systems

$$
\begin{aligned}
\varepsilon \dot{\tilde{z}}_{i, f}(t)= & \left(A_{22}-\lambda_{i} B_{2} K_{2}\right) \tilde{z}_{i, f}(t), \\
& \tilde{z}_{i, f}(0)=\tilde{z}_{i}(0)-\tilde{z}_{i, s}(0)
\end{aligned}
$$

where $K_{0}$ is the gain of the state feedback control that stabilizes the reduced order systems and

$$
A_{0}=A_{11}-A_{12} A_{22}^{-1} A_{21}, \quad B_{0}=B_{1}-A_{12} A_{22}^{-1} B_{2} .
$$

Throughout the rest of the paper, the following assumption is imposed.

Assumption 3: The pairs $\left(A_{0}, B_{0}\right)$ and $\left(A_{22}, B_{2}\right)$ are stabilizable.

Our main result in [11] is the following:

Theorem 2: Under Assumption 3, there exist $K_{2}$ and $K_{0}$ such that for $i=2, \ldots, n$ the matrices $A_{22}-\lambda_{i} B_{2} K_{2}$ and $A_{0}-\lambda_{i} B_{0} K_{0}$ are all Hurwitz. Then, there exists $\varepsilon^{*}>0$ such that the controllers (2) with

$$
K_{1}=\left(I_{m}-K_{2} A_{22}^{-1} B_{2}\right) K_{0}+K_{2} A_{22}^{-1} A_{21} .
$$

asymptotically synchronize with local information the systems (1).
In this paper, we enforce the state feedback simultaneous stabilization problem (SFSS) by considering the additional constraint that consists in ensuring a guaranteed cost on control effort.

Let us introduce $\widetilde{u}(t)=\left(T \otimes I_{m}\right) u(t)$ and notice that $\widetilde{u}(t)=\left(\widetilde{u}_{1}(t)^{\top}, \ldots, \widetilde{u}_{n}(t)^{\top}\right)^{\top}$ with

$$
\widetilde{u}_{i}(t)=-\lambda_{i} K_{1} \tilde{x}_{i}(t)-\lambda_{i} K_{2} \tilde{z}_{i}(t), \forall i \in 1, \ldots, n .
$$

Moreover, it is straightforward that

$$
\begin{aligned}
& u(t)^{\top}\left(I_{n} \otimes R\right) u(t)=\widetilde{u}(t)^{\top}\left(T T^{\top} \otimes R\right) \widetilde{u}(t) \\
& =\widetilde{u}(t)^{\top}\left(I_{n} \otimes R\right) \widetilde{u}(t)=\sum_{i=1}^{n} \widetilde{u}_{i}(t)^{\top} R \widetilde{u}_{i}(t)
\end{aligned}
$$

Remark 2: It is worth noting that using the change of variables (4) the global cost $J$ in (3) can be rewritten as a sum of individual costs associated with the systems in (5):

$$
\begin{aligned}
J & =\int_{0}^{\infty} \tilde{x}(t)^{\top}\left(D \otimes I_{n_{x}}\right) \tilde{x}(t)+\tilde{z}(t)^{\top}\left(D \otimes I_{n_{z}}\right) \tilde{z}(t) \\
& +\widetilde{u}(t)^{\top}\left(I_{n} \otimes R\right) \widetilde{u}(t) d t \\
& =\sum_{i=1}^{n}\left(\int_{0}^{\infty} \lambda_{i} \tilde{x}_{i}(t)^{\top} \tilde{x}_{i}(t)+\lambda_{i} \tilde{z}_{i}(t)^{\top} \tilde{z}_{i}(t)\right. \\
& \left.+\tilde{u}_{i}(t)^{\top} R \widetilde{u}_{i}(t) d t\right)=\sum_{i=1}^{n} \tilde{\mathcal{J}}_{i}
\end{aligned}
$$

where for all $i=1, \ldots, n$ one has

$\widetilde{\mathcal{J}}_{i}=\int_{0}^{\infty} \lambda_{i} \tilde{x}_{i}(t)^{\top} \tilde{x}_{i}(t)+\lambda_{i} \tilde{z}_{i}(t)^{\top} \tilde{z}_{i}(t)+\widetilde{u}_{i}(t)^{\top} R \widetilde{u}_{i}(t) d t$.

\section{B. Decentralized guaranteed cost control design}

Linear singularly perturbed systems in (5) can be rewritten as:

$$
\begin{aligned}
\left(\begin{array}{c}
\dot{\tilde{x}}_{i}(t) \\
\dot{\tilde{z}}_{i}(t)
\end{array}\right) & =\left(\begin{array}{cc}
A_{11} & A_{12} \\
\varepsilon^{-1} A_{21} & \varepsilon^{-1} A_{22}
\end{array}\right)\left(\begin{array}{c}
\tilde{x}_{i}(t) \\
\tilde{z}_{i}(t)
\end{array}\right) \\
& +\left(\begin{array}{c}
B_{1} \\
\varepsilon^{-1} B_{2}
\end{array}\right) \widetilde{u}_{i}(t), \quad i=1, \ldots, n
\end{aligned}
$$

where $\varepsilon>0, \tilde{x}_{i}(t) \in \mathbb{R}^{n_{x}}, \tilde{z}_{i}(t) \in \mathbb{R}^{n_{z}}$ are the components of the state variables defined in (4) and $\widetilde{u}_{i} \in \mathbb{R}^{m}$ is the control input defined in (6).

We recall that the synchronization problem of systems in (1) is translated into a simultaneous stabilization problem of systems in (5) or equivalently in (9). Furthermore, as shown in (7), the global cost associated with the asymptotic synchronization of the $n$ singularly perturbed systems (1) can be seen as the sum of $n$ individual costs associated with the stabilization of systems (5).

Remark 3: Since $\lambda_{1}=0$ it is noteworthy that $\widetilde{\mathcal{J}}_{1}=0$. In the following, for all $i=2, \ldots, n$, we rewrite $\widetilde{\mathcal{J}}_{i}$ more compactly as:

$$
\widetilde{\mathcal{J}}_{i}=\int_{0}^{\infty}\left(\lambda_{i} \widetilde{\mathbf{x}}_{i}(t)^{\top} \widetilde{\mathbf{x}}_{i}(t)+\widetilde{u}_{i}(t)^{\top} R \widetilde{u}_{i}(t)\right) d t
$$


where $\tilde{\mathbf{x}}_{i}(t)=\left[\tilde{x}_{i}(t)^{\top}, \tilde{z}_{i}(t)^{\top}\right]^{\top} \in \mathbb{R}^{n_{\mathbf{x}}=n_{x}+n_{z}}$ and the control input weight matrix $R \in \mathbb{R}^{m \times m}$ is symmetric positive definite.

Definition 3: We say that $\beta_{i}$ is a guaranteed cost for the $i^{\text {th }}$ system in (9) with the control law $\widetilde{u}_{i}(t)$ if the value of the cost function (10) satisfies the inequality $\widetilde{\mathcal{J}}_{i} \leqslant \beta_{i}$.

Remark 4: If there exists a guaranteed cost $\beta_{i}>0$ such that the closed-loop value of the cost function (10) satisfies $\widetilde{\mathcal{J}}_{i} \leqslant \beta_{i}$ for all $i=2, \ldots, n$ then a guaranteed cost $\bar{J} \triangleq(n-$ 1) $\max _{i=2}\left(\beta_{i}\right)$ is ensured for the global control performance required to asymptotically synchronize the collective closed loop dynamics (1).

It is noteworthy that the controllers in (6), which are used in Remark 4 above requires knowledge on the Laplacian eigenvalues. This means that the designed controller cannot be decentralized although it can be chosen to minimize the global cost function $J$ in (3). However, according to Assumption 1, the only available graph information consists in its connectivity.

In the following, we propose a decentralized guaranteed cost control law that simultaneously stabilizes the closedloop singularly perturbed uncertain systems (9) with respect to an adequate level of performance fixed by an upper bound on the integral cost function (10). Before providing the control design, let us introduce the following partitioned matrices

$$
A_{\varepsilon}=\left(\begin{array}{cc}
A_{11} & A_{12} \\
\varepsilon^{-1} A_{21} & \varepsilon^{-1} A_{22}
\end{array}\right), \quad B_{\varepsilon}=\left(\begin{array}{c}
B_{1} \\
\varepsilon^{-1} B_{2}
\end{array}\right)
$$

System (9) can be rewritten as follows :

$$
\dot{\widetilde{\mathbf{x}}}_{i}(t)=A_{\varepsilon} \widetilde{\mathbf{x}}_{i}(t)+B_{\varepsilon} \widetilde{u}_{i}(t), \quad \forall i=2, \ldots, n
$$

with the feedback control law $\widetilde{u}_{i}=F_{i} \hat{u}_{i}$ defined by $\hat{u}_{i}$ of the form

$$
\hat{u}_{i}(t)=-\mathcal{K} \widetilde{\mathbf{x}}_{i}(t)
$$

where $\mathcal{K}=\left[K_{1}, K_{2}\right] \in \mathbb{R}^{m \times n_{\mathrm{x}}}$, and $F_{i} \in R^{n_{\mathbf{x}} \times n_{\mathrm{x}}}$ defined as

$$
F_{i}=\lambda_{i} I_{n_{\mathbf{x}}}
$$

From basic properties of the Laplacian matrix (Remark 1), one can conclude that, for a given undirected graph of $n$ vertices, the following holds :

$$
\left(\lambda^{*}\right)^{2} I_{n_{\mathbf{x}}} \leqslant F_{i}^{\top} F_{i} \leqslant\left(\lambda^{\circ}\right)^{2} I_{n_{\mathbf{x}}}, i=2, \ldots, n
$$

Therefore, a general manner of studying (11) without the knowledge of $F_{i}$ is to analyse the system

$$
\dot{\tilde{\mathbf{x}}}(t)=A_{\varepsilon} \widetilde{\mathbf{x}}(t)+B_{\varepsilon} F \hat{u}(t),
$$

with $F$ an uncertain matrix satisfying (14). It is noteworthy that (14) is so called "norm bounded uncertainty" in robust control literature (see [14], [15]).

Remark 5: [9] Under Assumption 3, there exists $\varepsilon^{*}>0$ such that for all $\varepsilon \in\left(0, \varepsilon^{*}\right]$, the pair $\left(A_{\varepsilon}, B_{\varepsilon}\right)$ is stabilizable.

Theorem 4: Consider the uncertain system (15) and suppose Assumptions 1 and 3 hold. Then, there exists $\varepsilon^{*}>0$ such that for each $\varepsilon \in\left(0, \varepsilon^{*}\right]$ the following Riccati equation:

$$
P_{\varepsilon} A_{\varepsilon}+A_{\varepsilon}^{\top} P_{\varepsilon}-2\left(\lambda^{*}\right)^{2} P_{\varepsilon} B_{\varepsilon} R^{-1} B_{\varepsilon}^{\top} P_{\varepsilon}+\lambda^{\circ} I_{n_{\mathbf{x}}}=0
$$

admits a positive definite symmetric solution $P_{\varepsilon}$. Moreover, the controller

$$
\widetilde{u}_{i}(t)=-\lambda^{*} R^{-1} B_{\varepsilon}^{\top} P_{\varepsilon} \widetilde{\mathbf{x}}_{i}(t), \quad \forall i \in 2, \ldots, n
$$

stabilizes (11). Furthermore, a guaranteed cost $\beta_{i}=$ $\widetilde{\mathbf{x}}_{i}(0)^{\top} P_{\varepsilon} \widetilde{\mathbf{x}}_{i}(0)$ is achieved for the $i^{\text {th }}$ system in (11) using the controller (17).

Remark 6: The individual cost functions $\widetilde{\mathcal{J}}_{i}$ in (10) depend on $\lambda_{i}$ but neither the individual guaranteed costs $\beta_{i}$ nor the global guaranteed cost $\bar{J}$ does not depend on $\lambda_{i}$. This means that both the decentralized controllers (17) and the guaranteed cost are independent of $\lambda_{i}$ and they depend only on the network size: $n$.

Now our problem is stated as finding the matrix $P_{\varepsilon}$ which through conditions in Theorem 4 , determines the solution to the guaranteed cost control problem for the $i^{\text {th }}$ singularly perturbed system. Indeed, the Riccati equation (16) is first solved for $P_{\varepsilon}$ and then the guaranteed cost controller gains are obtained by substituting the Riccati solution to (17).

Remark 7: Note that the obtained cost $\beta_{i}$ in Theorem 4 depends on the initial conditions $\tilde{x}_{i}(0)$ and $\tilde{z}_{i}(0)$. This dependance can be removed by assuming that initial conditions are zero mean random variables with $E\left[\widetilde{\mathbf{x}}_{i}(0) \widetilde{\mathbf{x}}_{i}(0)^{\top}\right]=I_{n_{\mathbf{x}}}$.

\section{Numerical implementation for decentralized guaranteed cost control design}

It is important to stress that some numerical problems may arise in solving equation (16) because of the different magnitudes of its coefficients caused by the small parameter $\varepsilon$. Inspired by [16] we provide in this subsection an approach that overcomes these computation difficulties. The idea is to transform the full-order Riccati equation (11) depending on $\varepsilon$ into slow and fast Riccati equations independent of $\varepsilon$. Doing so, we find a stabilizing solution for the guaranteed cost control problem of singularly perturbed uncertain system (15).

First, let us introduce the following lemma that will be used to solve the algebraic Riccati equation (16).

Lemma 5: The algebraic Riccati equation (16) is equivalent to the following Riccati equation :

$$
P A+A^{\top} P-2\left(\lambda^{*}\right)^{2} P B R^{-1} B^{\top} P+\lambda^{\circ} I_{n_{\mathbf{x}}}=0
$$

where $P=\Gamma_{\varepsilon}^{-1} P_{\varepsilon}, A=\Gamma_{\varepsilon} A_{\varepsilon}, B=\Gamma_{\varepsilon} B_{\varepsilon}$,

$$
\begin{aligned}
& P_{\varepsilon}=\left(\begin{array}{cc}
P_{11} & \varepsilon P_{21}^{\top} \\
\varepsilon P_{21} & \varepsilon P_{22}
\end{array}\right), \text { such that }\left\{\begin{array}{l}
P_{11}=P_{11}^{\top} \\
P_{22}=P_{22}^{\top}
\end{array}\right. \\
& \Gamma_{\varepsilon}=\left(\begin{array}{cc}
I_{n_{x}} & 0_{n_{x}, n_{z}} \\
0_{n_{z}, n_{x}} & \varepsilon I_{n_{z}}
\end{array}\right), \text { for all } \varepsilon \in\left(0, \varepsilon^{*}\right) .
\end{aligned}
$$

Further, by making use of relation (19), the controller gain in (17) becomes

$$
\mathcal{K}=\left[K_{1}, K_{2}\right]=R^{-1} B^{\top} P .
$$

It is worth noting that $P_{\varepsilon}=\Gamma_{\varepsilon}^{\top} P=P^{\top} \Gamma_{\varepsilon}$ is symmetric but $P$ is not. 
The Riccati equation (18) can be partitioned into

$$
\begin{aligned}
& P_{11} A_{11}+A_{11}^{\top} P_{11}+P_{21}^{\top} A_{21}+A_{21}^{\top} P_{21}-P_{11} \widetilde{S}_{11} P_{11} \\
& -P_{21}^{\top} \widetilde{S}_{12}^{\top} P_{11}-P_{11} \widetilde{S}_{12} P_{21}-P_{21}^{\top} \widetilde{S}_{22} P_{21}+\lambda^{\circ} I_{n_{x}}=0 \\
& \varepsilon P_{21} A_{11}+P_{22} A_{21}+A_{12}^{\top} P_{11}+A_{22}^{\top} P_{21}-\varepsilon P_{21} \widetilde{S}_{11} P_{11} \\
& -\varepsilon P_{21} \widetilde{S}_{12} P_{21}-P_{22} \widetilde{S}_{12} P_{11}-P_{22} \widetilde{S}_{22} P_{21}=0 \\
& \varepsilon P_{21} A_{12}+\varepsilon A_{12}^{\top} P_{21}^{\top}+P_{22} A_{22}+A_{22}^{\top} P_{22}-\varepsilon^{2} P_{21} \widetilde{S}_{11} P_{21}^{\top} \\
& -\varepsilon P_{22}^{\top} \widetilde{S}_{12}^{\top} P_{21}^{\top}-\varepsilon P_{21} \widetilde{S}_{12} P_{22}-P_{22} \widetilde{S}_{22} P_{22}+\lambda^{\circ} I_{n_{z}}=0
\end{aligned}
$$

where

$$
\widetilde{S}=2\left(\lambda^{*}\right)^{2} B R^{-1} B^{\top}=\left(\begin{array}{cc}
\widetilde{S}_{11} & \widetilde{S}_{12} \\
\widetilde{S}_{12}^{\top} & \widetilde{S}_{22}
\end{array}\right) .
$$

Similar to Theorem 2 in [16], we apply the implicit function theorem to show the existence of a bounded solution $P$ in neighborhood of $\varepsilon=0$.

Setting $\varepsilon \rightarrow 0$ in equations (21)-(23), one obtains :

$$
\begin{aligned}
& \bar{P}_{11}^{\top} A_{11}+A_{11}^{\top} \bar{P}_{11}+\bar{P}_{21}^{\top} A_{21}+A_{21}^{\top} \bar{P}_{21}-\bar{P}_{11} \widetilde{S}_{11} \bar{P}_{11} \\
& -\bar{P}_{21}^{\top} \widetilde{S}_{12}^{\top} \bar{P}_{11}-\bar{P}_{11} \widetilde{S}_{12} \bar{P}_{21}-\bar{P}_{21}^{\top} \widetilde{S}_{22} \bar{P}_{21}+\lambda^{\circ} I_{n_{x}}=0 \\
& \bar{P}_{22}^{\top} A_{21}+A_{12}^{\top} \bar{P}_{11}+A_{22}^{\top} \bar{P}_{21}-P_{22}^{\top} \widetilde{S}_{12} \bar{P}_{11}-\bar{P}_{22} \widetilde{S}_{22} \bar{P}_{21} \\
& =0 \\
& \bar{P}_{22}^{\top} A_{22}+A_{22}^{\top} \bar{P}_{22}-\bar{P}_{22} \widetilde{S}_{22} \bar{P}_{22}+\lambda^{\circ} I_{n_{z}}=0
\end{aligned}
$$

where $\bar{P}_{11}, \bar{P}_{21}, \bar{P}_{22}$ are the limiting solutions when $\varepsilon \rightarrow 0$.

Let us define the following set :

$\mathcal{L}_{f}=\{n>1$, such that the Riccati equation (26) has a

positive definite solution $\}$,

Note that $A_{22}-\widetilde{S}_{22} \bar{P}_{22}$ is non-singular. Thus, equations (24)-(26) become as follows :

$$
\begin{aligned}
& \bar{P}_{11} \bar{A}_{0}+\bar{A}_{0}^{\top} \bar{P}_{11}-\bar{P}_{11} \widetilde{S}_{0} \bar{P}_{11}+\widetilde{Q}_{0}=0 \\
& \bar{P}_{21}=-N_{2}^{\top}+N_{1}^{\top} \bar{P}_{11} \\
& \bar{P}_{22} A_{22}+A_{22}^{\top} \bar{P}_{22}-\bar{P}_{22} \widetilde{S}_{22} \bar{P}_{22}+\lambda^{\circ} I_{n_{z}}=0
\end{aligned}
$$

where

$$
\begin{aligned}
& N_{1}=-D_{1} D_{2}^{-1}, \quad N_{2}=A_{21}^{\top} \bar{P}_{22} D_{2}^{-1} \\
& \widetilde{Q}_{0}=n I_{n_{x}}-N_{2} A_{21}-A_{21}^{\top} N_{2}^{\top}-N_{2} \widetilde{S}_{22} N_{2}^{\top}
\end{aligned}
$$

and

$$
\begin{aligned}
\bar{A}_{0} & =A_{11}+N_{1} A_{21}+\widetilde{S}_{12} N_{2}^{\top}+N_{1} \widetilde{S}_{22} N_{2}^{\top} \\
\widetilde{S}_{0} & =\widetilde{S}_{11}+N_{1} \widetilde{S}_{12}^{\top}+\widetilde{S}_{12} N_{1}^{\top}+N_{1} \widetilde{S}_{22} N_{1}^{\top} \\
D_{1} & =A_{12}-\widetilde{S}_{12} \bar{P}_{22} \\
D_{2} & =A_{22}-\widetilde{S}_{22} \bar{P}_{22} .
\end{aligned}
$$

Let us also introduce :

$$
\mathcal{L}_{s}=\{n>1 \text {, such that the Riccati equation (27) has a }
$$

$$
\text { positive definite solution\}. }
$$

Summarizing, instead of solving (16) we can approximate its solution by solving (18) as stated in the next theorem.
Theorem 6: For all $n \in \mathcal{L}$, there exists $0<\bar{\varepsilon}<\varepsilon^{*}$ such that for all $\varepsilon \in(0, \bar{\varepsilon})$, the generalized algebraic Riccati equation (18) has a positive definite solution of the form

$$
P=\left(\begin{array}{cc}
\bar{P}_{11}+\mathcal{O}(\varepsilon) & \varepsilon \bar{P}_{12}^{\top}+\mathcal{O}\left(\varepsilon^{2}\right) \\
\bar{P}_{21}+\mathcal{O}(\varepsilon) & \bar{P}_{22}+\mathcal{O}(\varepsilon)
\end{array}\right)
$$

where $\mathcal{L}=\mathcal{L}_{s} \bigcap \mathcal{L}_{f}$. Thus, a guaranteed cost controller is given by (17) with $P_{\varepsilon}=\Gamma_{\varepsilon}^{\top} P$ is a positive definite matrix solving the Riccati equation (16).

Proof: The proof follows by combining Theorem 4 and Lemma 5.

Theorem 6 implies that, if the Riccati equation (18) has a positive matrix solution $P$ then the solution $P_{\varepsilon}$ of (16) will be the smallest upper bound on the criterion (10).

Further, the controller (17) guarantees a simultaneous stabilization of the $n-1$ subsystems (11) with a guaranteed cost of value $\beta_{i}$. Consequently, the $n$ singularly perturbed systems (1) achieve asymptotic synchronization under the state feedback controller (2) with the matrix gain (20). In addition, the global control effort required to achieve the synchronization is upper-bounded by $\bar{J}=(n-1) \max _{i=2, \ldots, n}\left(\beta_{i}\right)$.

\section{NUMERICAL EXAMPLES}

In this section, we consider the synchronization of three agents whose dynamics are given by (1) where :

$$
\begin{aligned}
& A_{11}=\left(\begin{array}{cc}
2.5 & -6 \\
-2 & 2
\end{array}\right), A_{12}=\left(\begin{array}{cc}
2 & 3 \\
0 & -2
\end{array}\right), B_{1}=\left(\begin{array}{l}
2 \\
1
\end{array}\right) \\
& A_{21}=\left(\begin{array}{ll}
0.5 & 2 \\
-1 & 1
\end{array}\right), A_{22}=\left(\begin{array}{cc}
-2 & 1 \\
0 & -1
\end{array}\right), B_{2}=\left(\begin{array}{l}
1 \\
1
\end{array}\right) .
\end{aligned}
$$

To each agent we assign a vector state having 4 components characterized by slow and fast dynamics.

For any agent $i \in\{1,2,3\}$, let us denote by $\left[x_{i, 1}, x_{i, 2}\right]^{\top}$ and $\left[z_{i, 1}, z_{i, 2}\right]^{\top}$ its slow and fast state' components, respectively.

The communication network among the 3 agents is described by an undirected graph $\mathcal{G}$ which is connected and the following Laplacian matrix :

$$
L=\left(\begin{array}{ccc}
3 & -1 & -2 \\
-1 & 3 & -2 \\
-2 & -2 & 4
\end{array}\right)
$$

The bounds on the eigenvalues of $L$ are $\lambda^{*}=0.0278$ and $\lambda^{\circ}=6$.

In simulation we fix $\varepsilon=0.01$ and the components of the initial condition are chosen for the 3 agents as $[2.5,2,-0.5,-1.5],[1.5,1,4,-2],[0.5,-1,3,1]$. Note that, when rewriting the system dynamics as in (11), one can verify that Assumption 3 holds and the uncertainties are given by $F_{i=2,3} \in\left\{4 \times I_{4}, 6 \times I_{4}\right\}$. We choose $R=1$ in (10) for the cost function. To apply Theorem 4 one needs to compute the matrix $P_{\varepsilon}$. In order to do that we first solve (18) to obtain

$$
P=\left(\begin{array}{cccc}
5.7472 & -2.6140 & 0.0578 & 0.2431 \\
-2.6140 & 132.3560 & -0.0061 & -2.6695 \\
5.7762 & -0.6120 & 1.5555 & 0.7322 \\
24.3125 & -266.9538 & 0.7322 & 9.7476
\end{array}\right)
$$


Using Theorem 6 we compute

$$
P_{\varepsilon}=\left(\begin{array}{cccc}
5.7472 & -2.6140 & 0.0578 & 0.2431 \\
-2.6140 & 132.3560 & -0.0061 & -2.6695 \\
0.0578 & -0.0061 & 0.0156 & 0.0073 \\
0.2431 & -2.6695 & 0.0073 & 0.0975
\end{array}\right) .
$$

which yields the gain matrix

$$
\mathcal{K}=\left[\begin{array}{ll}
K_{1} & K_{2}
\end{array}\right]=[38.96,-140.43,2.4,8.29] .
$$

Figures (1) and (2) highlight the simultaneous synchronization of the slow and fast dynamics.

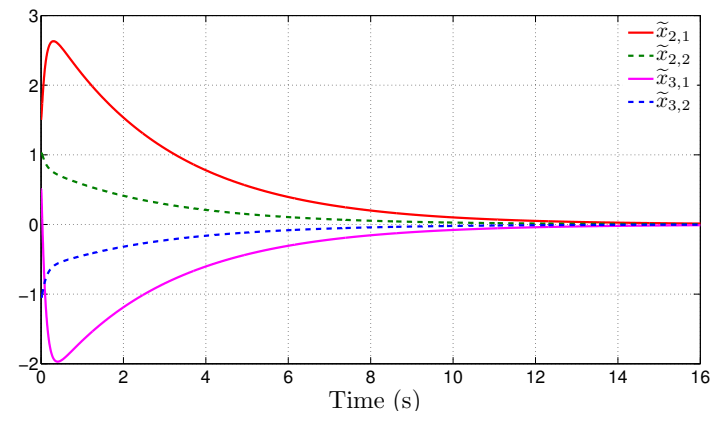

Fig. 1: The trajectories of $\tilde{x}$

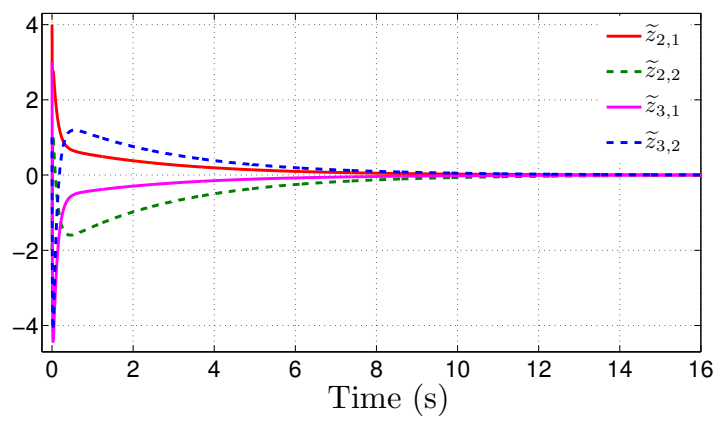

Fig. 2: The trajectories of $\tilde{z}$

Next, we apply the control law in (2) with the obtained gain matrix $\mathcal{K}$ to the two time scale model (1).
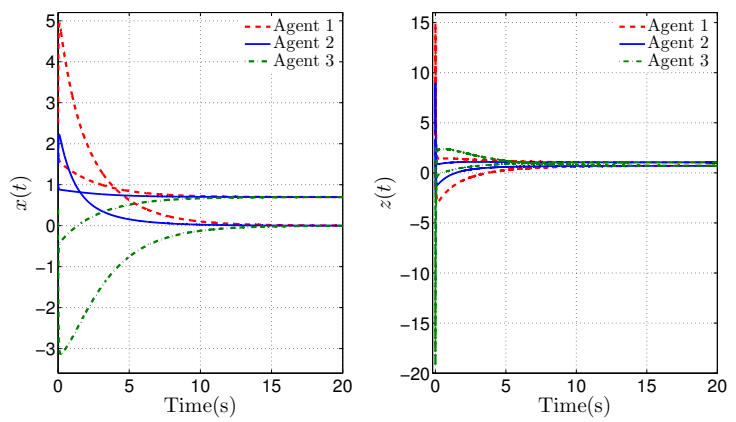

Fig. 3: State trajectories of the system

From Fig 3, it can be observed that the states of the closed-loop systems reach consensus with a global guaranteed cost $\bar{J}=295.66$.

\section{CONCLUSion}

In this paper we propose a decentralized control strategy that allows singularly perturbed multi-agent systems to achieve synchronization with global performance guarantees. The main difficulty that we have to overcome is that we have to avoid the use of centralized information related to the interconnection network structure. This problem is solved by sequentially rewriting the synchronization problem in terms of simultaneous stabilization of singularly perturbed systems and then as stabilization of a singularly perturbed uncertain linear system. The singularly perturbed dynamics of subsystems generates theoretical challenges related to the stabilizing controller design but also numerical issues related to the computation of the controller gains. We show that these problems can be solved by decoupling the slow and fast dynamics.

\section{REFERENCES}

[1] F. Bullo, J. Cortés, and S. Martinez, Distributed Control of Robotic Networks. A Mathematical Approach to Motion Coordination Algorithms. Princeton University Press, 2009.

[2] V. D. Blondel, J. M. Hendrickx, , and J. Tsitsiklis, "On Krause's multi-agent consensus model with state-dependent connectivity," IEEE Trans. on Automatic Control, vol. 54, no. 11, pp. 2586-2597, 2009.

[3] I.-C. Morărescu and A. Girard, "Opinion dynamics with decaying confidence: Application to community detection in graphs," IEEE Trans. on Automatic Control, vol. 56, no. 8, pp. 1862 - 1873, 2011.

[4] I.-C. Morărescu, W. Michiels, and M. Jungers, "Synchronization of coupled nonlinearoscillators with shifted gamma-distributed delays," in Proceedings of American Control Conference, 2013.

[5] L. Buşoniu and I.-C. Morărescu, "Consensus for black-box nonlinear agents using optimistic optimization," Automatica, vol. 50, no. 4, pp. 1201-1208, 2014.

[6] L.Chen and K. Aihara, "A model of periodic oscillation for genetic regulatory systems," IEEE Transactions on Circuits and Systems, vol. 49, no. 10, pp. 1429-1436, 2002.

[7] A. Hodgkin and A. Huxley, "A quantitative description of membrane current and its application to conduction and excitation in nerve," The Journal of Physiology, vol. 117, pp. 500-544, 1952.

[8] I. Malloci, J. Daafouz, and C. Iung, "Stabilization of continuous-time singularly perturbed switched systems," in Proc. of the 48th IEEE Conference on Decision and Control, 2009.

[9] P. Kokotović, H. Khalil, and J. O'Reilly, Singular perturbation methods in control: analysis and design. SIAM Series in Classics and Applied Mathematics, 1999.

[10] J. Hassan and J. Shamma, "Decentralized energy aware cooptimization of mobility and communication in multiagent systems," in Proceedings of 55th IEEE Conference on Decision and Control, CDC 2016, 2016

[11] J. B. Rejeb, I. C. Morărescu, and J. Daafouz, "Synchronization in networks of linear singularly perturbed systems," in American Control Conference, July 2016, pp. 4293-4298.

[12] C. Godsil and G. Royle, Algebraic Graph Theory. Springer-Verlag, New-York, 2001.

[13] S. Friedland and R. Nabben, "On cheeger-type inequalities for weighted graphs," Journal of Graph Theory, vol. 41, no. 1, pp. 117, 2002.

[14] H. Mukaidani and K. Mizukami, "The guaranteed cost control problem of uncertain singularly perturbed systems," Journal of Mathematical Analysis and Applications, vol. 251, no. 2, pp. 716 - 735, 2000.

[15] G. Garcia, J. Daafouz, and J. Bernussou, " $\mathrm{H}_{2}$ guaranteed cost control for singularly perturbed uncertain systems," IEEE Transactions on Automatic Control, vol. 43, no. 9, pp. 1323-1329, Sep 1998.

[16] H. Mukaidani, H. Xu, and T. Okita, "Robust stabilization of nonstandard singularly perturbed systems with uncertainties," in IFAC World Congress, G, vol. 151, 1999, p. 156. 